\title{
Rheology of soft granular materials: uniaxial compression
}

\author{
Saeid Nezamabadi ${ }^{1,2,3, *}$, Farhang Radjai ${ }^{1}$, Serge Mora ${ }^{1}$, Jean-Yves Delenne ${ }^{2}$, and Mojtaba Ghadiri ${ }^{3}$ \\ ${ }^{1}$ LMGC, Univ. Montpellier, CNRS, Montpellier, France \\ ${ }^{2}$ IATE, Univ. Montpellier, INRAE, Montpellier SupAgro, Montpellier, France \\ ${ }^{3}$ School of Chemical and Process Engineering, University of Leeds, Leeds LS2 9JT, UK
}

\begin{abstract}
Soft granular materials are assemblies of highly deformable grains interacting via surface forces The large grain deformations of these materials differ them from hard granular systems, in which, their behaviors are essentially governed by grain rearrangements. In this paper, we study the uniaxial compression of soft granular materials using a numerical approach based on the Material Point Method allowing for large grain deformations, coupled with the Contact Dynamics method for the treatment of unilateral frictional contacts between grains. Considering the neo-Hookean and elasto-plastic grains, the compaction of 2D soft granular packings is analyzed. We focus essentially on the evolution of the packing vertical stress as a function of the packing fraction and the predictive models are proposed.
\end{abstract}

\section{Introduction}

Soft granular materials such as metallic powders and, many pharmaceutical and food products can deform elastically or plastically without rupture under low confining pressure. It allows them to achieve high packing fractions above the jamming state of hard granular materials. In the context of soft granular materials, a combination of grain rearrangements and grain shape changes hence controls their rheological properties contrary to hard granular materials [1-4].

In our previous works, we proposed three numerical approaches to study the rheology of soft granular materials. First method is the Bonded Particle Model (BPM), which consists in modeling each grain as an aggregate composed of hard primary particles with cohesive interactions such that a grain can deform as a result of the relative motions of the particles while staying together as a solid grain [5]. Two other approches are based on a combination of the continuum approaches (the Material Point Method (MPM) and the Finite Element Method (FEM)), accounting for a broad class of mechanical behaviors (elastic, plastic...) of individual grains to deal with grain deformations, and the Contact Dynamics (CD) method to treat contact interactions between grains [5-8].

In this work, we investigate the compaction of neoHookean and elasto-plastic grain packings using the MPM-CD approach. The rheology of the soft granular systems by considering several parameters (stress, packing fraction, connectivity...) are investigated around and beyond the jamming or Random Close Packing (RCP) state (i.e., where it is difficult for grains to move individually). We will highlight the influence of grain shape change on space filling properties, stress transmission and texture be-

*e-mail: saeid.nezamabadi@umontpellier

A video is available at https://doi.org/10.48448/jnd0-h256

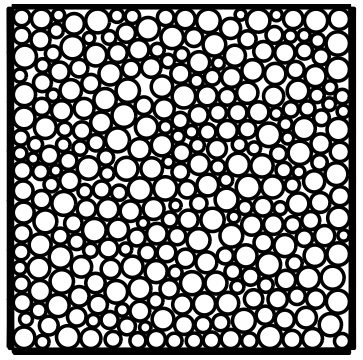

(a) Initial state: $\Phi=0.78$

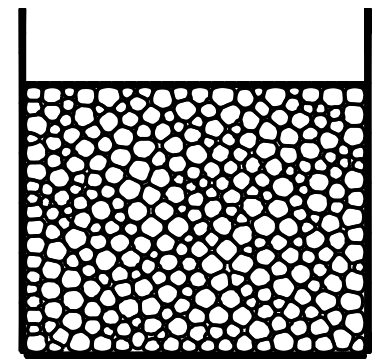

(b) $\Phi=0.97$
Figure 1. Snapshots of the initial configuration (a), and of the compaction of an elastic grain packing for packing fraction of $\Phi=0.97$ (b)

yond the RCP state. In particular, we will focus on the evolution of stress as a function of packing fraction.

\section{Around the jamming state}

The compaction of soft granular materials are governed by three parameters: (i) grain rearrangements; (ii) grain shape changes, and (iii) grain deformations. The rearrangement of grains are occurred essentially before the jamming state. Beyond the jamming state, grain shape and volume changes are controlled the packing evolutions and allow to the packing fraction to exceed the one corresponding to the RCP state.

We consider the uniaxial compression of a packing of 300 elastic grains (see Sec. 3.2). In Fig. 1, we observe that the grain shapes change from circular to become almost polygonal and the grain deformations lead to a reduction in pore size between the grains. Fig. 2 shows the vertical 


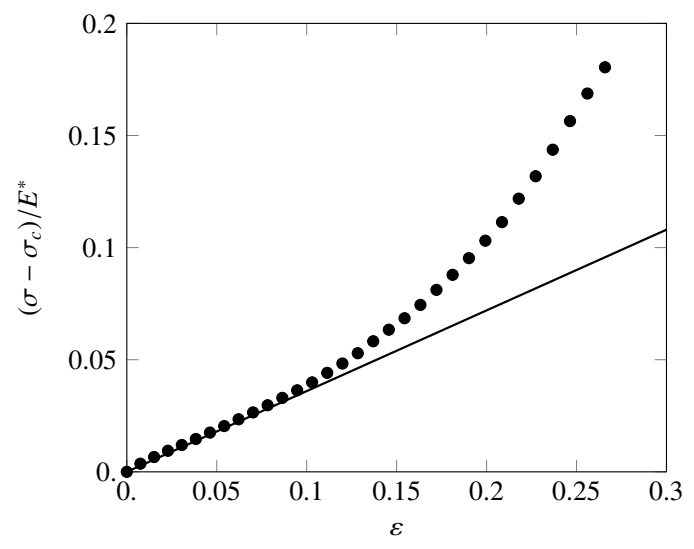

Figure 2. The excess vertical stress $\sigma-\sigma_{c}$ normalized by the effective modulus $E^{*}$ as a function of the vertical strain $\varepsilon$ for an elastic grain packing. $\sigma_{c}$ correspond to the jamming point; here, $\Phi_{c} \simeq 0.8$. The lines show the predicted behavior around the jamming state; see Eq. (1).

stress $\sigma$ as a function of the cumulative vertical strain $\varepsilon$ beyond the jamming state for this packing. We observe a linear behavior near the jamming state due to small deformation of grains. Indeed, around the jamming state, the grain shape and volume changes are negligible and the soft granular packing behaves almost like a hard granular medium with a Hertz's contact law. We can thus consider in $2 \mathrm{D}[5,9]$ :

$$
\begin{aligned}
& \sigma=\frac{Z \Phi}{\pi<d>}<f_{n}>, \\
& <f_{n}>=\frac{\pi}{8} E^{*}<d>\varepsilon,
\end{aligned}
$$

where $Z$ and $\Phi$ are, respectively, the coordination number and the packing fraction $\left(\Phi=V_{S} / V\right.$, where $V_{S}$ is the volume of grains and $V$ the total volume). $<d>$ denotes the mean diameter of grains. $<f_{n}>$ represents the mean normal contact force between grains and $E^{*}$ is the effective modulus: $E^{*}=E /\left(1-v^{2}\right)$. By replacing Eq. (2) into Eq. (1), one can obtain:

$$
\sigma=\frac{Z \Phi}{8} E^{*} \varepsilon .
$$

Given that around the jamming state, in $2 \mathrm{D}, Z \simeq 4$ and $\Phi \simeq 0.8$, we get: $\sigma \simeq 0.4 E^{*} \varepsilon$. In Fig. 2 , we can observe this linear behavior with a slope a little less than 0.4. Here, this difference can be explained by the grain discretization effects. Indeed, the value of $E^{*}$, depending on the discretization, is a little larger than the analytical value and increases as the fineness of discretization increases [6]. Note that this linear behavior near the jamming sate, can be seen for all types of grain material behavior. However, beyond the jamming state, a non-linear behavior is observed due to the grain shape and volume changes as shown in Fig. 2. This behavior will be studied in the following.

\section{Beyond the jamming state}

Grains start to undergo large deformations beyond the jamming state. This leads to an increasing number of contacts and increase in the size of contacts between grains and thus, filling the gaps and inter-grain porosities. So, the packing can achieve high values of packing fraction. To study the packing behavior beyond the jamming state, two grain behaviors are considered: neo-Hookean and elastoplastic.

\subsection{Neo-Hookean grain packings}

We investigate first the compaction of 300 neo-Hookean grains by means of MPM simulations. The preparation of the initial configuration is carried out using DEM simulations. We introduce a small size polydispersity in order to avoid long-range ordering (diameters $\in[2,4] \mathrm{mm}$ ). The gravitational acceleration and friction between the grains, and between the grains and the walls of the confining box are set to be zero. The top wall is moved down at constant velocity of $2 \mathrm{~m} / \mathrm{s}$ with a time step of $\delta t=0.1 \mu \mathrm{s}$ to simulate uniaxial compression. The MPM simulations are performed in $2 \mathrm{D}$ in plane strain conditions. The constitute law of neo-Hookean grains are [7]:

$$
\mathbf{S}=(\lambda \ln (J)-\mu) \mathbf{C}^{-1}+\mu \mathbf{I},
$$

where $\mathbf{S}$ and $\mathbf{C}$ is the second Piola-Kirchhoff stress tensor and the right Cauchy-Green strain tensor, respectively. $\mathbf{C}$ is related to the deformation gradient tensor, $\mathbf{F}$, through $\mathbf{C}=\mathbf{F}^{T} \mathbf{F}$ and $J=\operatorname{det}(\mathbf{F}) . \quad \lambda$ and $\mu$ are the Lamé's parametrs, and $\mu$ also represents the shear modulus. In our simulations, we set the density of the grains $\rho=990$ $\mathrm{kg} / \mathrm{m}^{3}$ and $\mu=1.5 \mathrm{MPa}$. We consider three values of $\lambda$ to define the compressibility of the grain: $\lambda=0,3$ and 100 $\mathrm{MPa}$ (for $\lambda=0$, the grain is fully compressible whereas for $\lambda=100 \mathrm{MPa}$, the grain is quasi-incompressible).

Fig. 3 represents the excess vertical stress $\sigma-\sigma_{c}$ normalized by the grain bulk modulus $K\left(K=\lambda+\frac{2}{3} \mu\right)$ as a function of the packing fraction $\Phi$ beyond the jamming state. Here, the jamming point is considered to be $\Phi_{c} \simeq 0.8$ [7] and $\sigma_{c}$ corresponds to this point. Note that the value of $\sigma_{c}$ is small and our simulations are almost quasi-static. A nonlinear behavior is observed for all three cases at different rates.

To describe the evolution of the vertical stress as a function of the packing fraction, we can consider that the packing behaves like a porous media beyond jamming state since there is no rearrangement. So, the vertical stress $\sigma$ may be related to the vertical strain $\varepsilon$, via an effective Pwave modulus $M^{\text {eff }}$ :

$$
\sigma=M^{\mathrm{eff}} \varepsilon
$$

Wa can also assume that the grain bulk modulus $K$ relates the grain volume $V_{S}$ to the grain effective stress $\sigma_{S}$ :

$$
K \frac{d V_{s}}{V_{s}}=-d \sigma_{S}
$$

For a granular system, the stress $\sigma$ is proportional to the mean grain stress $\sigma_{S}$ via $Z$ and $\Phi: \sigma \propto Z \Phi \sigma_{S}$. Since also $d \varepsilon=d V_{S} / V_{S}-d \Phi / \Phi$, one can obtain the following 


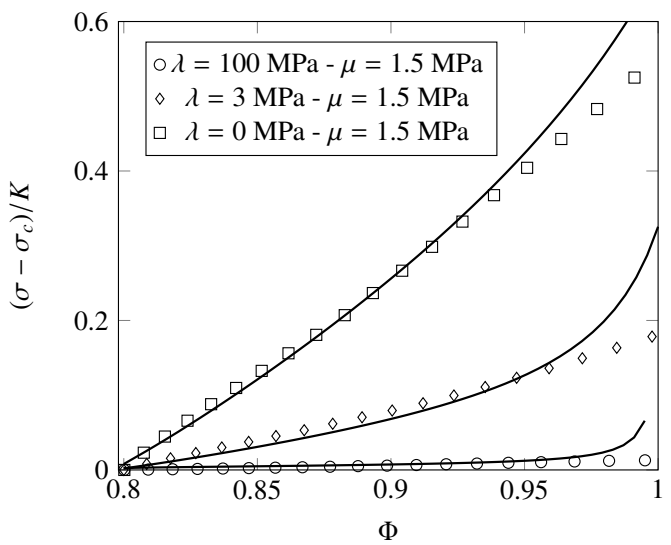

Figure 3. Evolution of the excess applied stress $\sigma-\sigma_{c}$ normalized by the grain bulk modulus $K$ as a function of packing fraction $\Phi$ for neo-Hookean grains. $\sigma_{c}$ corresponds to the jamming point, $\Phi_{c} \simeq 0.8$. The lines show the predicted behaviors beyond the jamming state by the compaction models introduced in Eq. (8).

differential equation to solve by using Eqs. (5) and (6):

$\left(1+\frac{M^{\text {eff }}}{c_{1} Z \Phi}\right) \mathrm{d} \sigma=\left[\frac{M^{\text {eff }}}{c_{1} Z \Phi}\left(\frac{\mathrm{d} \Phi}{\Phi}+\frac{\mathrm{d} Z}{Z}\right)+\frac{\mathrm{d} M^{\text {eff }}}{M^{\text {eff }}}\right] \sigma-M^{\text {eff }} \frac{\mathrm{d} \Phi}{\Phi}$,

where $c_{1}$ is a material constant to be determined. By knowing that $M^{\text {eff }}$ and $Z$ are related to $\Phi$ [7], the resolution of the differential equation (7) yields:

$$
\sigma=-\frac{1}{\frac{1}{M^{\mathrm{eff}}}+\frac{1}{c_{1} Z \Phi}}\left(\ln (\Phi)+c_{2}\right)
$$

where $c_{2}$ is the integration constant.

Since, as mentioned above, beyond jamming, the soft granular packing behaves almost like a porous material, the effective P-wave modulus $M^{\text {eff }}$ can be given by:

$$
M^{\mathrm{eff}}=\frac{\mu^{\mathrm{eff}}\left(4 \mu^{\mathrm{eff}}-E^{\mathrm{eff}}\right)}{3 \mu^{\mathrm{eff}}-E^{\mathrm{eff}}},
$$

where $E^{\text {eff }}$ is the effective Young's modulus defined by [10]:

$$
E^{\mathrm{eff}}=E\left(1-\frac{p}{p_{c}}\right)^{f_{E}},
$$

and the effective shear modulus is [11]:

$$
\mu^{\mathrm{eff}}=\mu\left(1-\frac{p}{p_{c}}\right)^{f_{\mu}} .
$$

Here, $p$ is the porosity $(p=1-\Phi), f_{E}$ and $f_{\mu}$ denote the characteristic components for Young's and shear moduli, and $p_{c}$ is the critical porosity, below which the effective Young's and shear moduli become zero. This corresponds to below and near the jamming point.

In Fig. 3, the predictions of the model (8) are presented with $c_{1}=100, f_{E}=1.1, f_{\mu}=0.1, p_{c}=0.201\left(\simeq 1-\Phi_{c}\right)$ and $c_{2}=0.23$ for $\lambda=0$ and $3 \mathrm{MPa}$, and $c_{2}=0.43$ for $\lambda=$ $100 \mathrm{MPa}$. These predictions are in good agreement with the MPM simulations and are shown that this analytical model reproduce well the numerical results. However, at high packing fractions, a divergence between numerical results and analytical predictions is observed. It is due to almost coarse discretization of grain boundaries.

\subsection{Elasto-plastic grain packings}

We carried out the MPM simulations of the same packing as before and with the same conditions by considering here two other types of constitutive laws: linear elastic and elasto-plastic with linear isotropic hardening [12]. For these two material behaviors, we set $E=10 \mathrm{MPa}, v=0.45$ (Poisson's ratio) and $\rho=1000 \mathrm{~kg} / \mathrm{m}^{3}$, and for the elastoplastic behavior, the yield stress is equal to $\sigma_{y}=0.4 \mathrm{MPa}$. The hardening is also set to $H=0,1$ and $3 \mathrm{MPa}$. Fig. 4 shows the vertical stress $\sigma$ as a function of the packing fraction $\Phi$ for elastic and elasto-plastic grains. We also observe a nonlinear behavior beyond the jamming state.

To describe the packing of elasto-plastic grains, the vertical stress $\sigma$ may be decomposed into an elastic part $\sigma_{e}$ and a plastic one $\sigma_{p}$ as follows:

$$
\sigma=\left(1-f_{p}\right) \sigma_{e}+f_{p} \sigma_{p},
$$

where $f_{p}$ is the plastic volume fraction defined as the ratio between the volume of the plastic regions of grains and their total volume. During the compaction process, $f_{p}$ is zero before the jamming, but beyond the jamming point, it is proportional to the vertical strain (hence, the packing fraction). One can therefore assume that $f_{p}$ increases logarithmically with $\Phi / \Phi_{c}$ :

$$
f_{p}=\alpha \ln \left(\frac{\Phi}{\Phi_{c}}\right),
$$

where $\alpha$ is a constant parameter to be determined. Moreover, the plastic stress $\sigma_{p}$ can be defined in the following form by considering Eq. (8):

$$
\sigma_{p}=\sigma_{y}-\frac{1}{\frac{1}{M_{p}^{\text {eft }}}+\frac{1}{c_{1_{p}} Z \Phi}}\left(\ln (\Phi)+c_{2_{p}}\right),
$$

with

$$
\begin{gathered}
M_{p}^{\mathrm{eff}}=\frac{\mu_{p}^{\mathrm{eff}}\left(4 \mu_{p}^{\mathrm{eff}}-E_{p}^{\mathrm{eff}}\right)}{3 \mu_{p}^{\mathrm{eff}}-E_{p}^{\mathrm{eff}}}, \\
E_{p}^{\mathrm{eff}}=E_{p}\left(1-\frac{p}{p_{c}}\right)^{f_{E_{p}}}, \\
\mu_{p}^{\mathrm{eff}}=\mu_{p}\left(1-\frac{p}{p_{c}}\right)^{f_{\mu_{p}}} .
\end{gathered}
$$

Here, $E_{p}$ is the plastic tangent modulus related to $H$ through: $E_{p}=E H /(E+H)$ and $\mu_{p}$ denotes the plastic shear modulus: $\mu_{p}=E_{p} / 2\left(1+v_{p}\right)$. Note that, by assuming that the volume change due to plastic deformation is negligible, one concludes the equality of the elastic and plastic Poisson's ratios; i.e. $v_{p}=v$.

We can see the predictions of the models (8) and (12) in Fig. 4 with $c_{1}=c_{1_{p}}=100, c_{2}=c_{2_{p}}=0.23, f_{E}=$ $f_{E_{p}}=1.1, f_{\mu}=f_{\mu_{p}}=0.1, p_{c}=0.201$ and $\alpha=2.5$. It is 


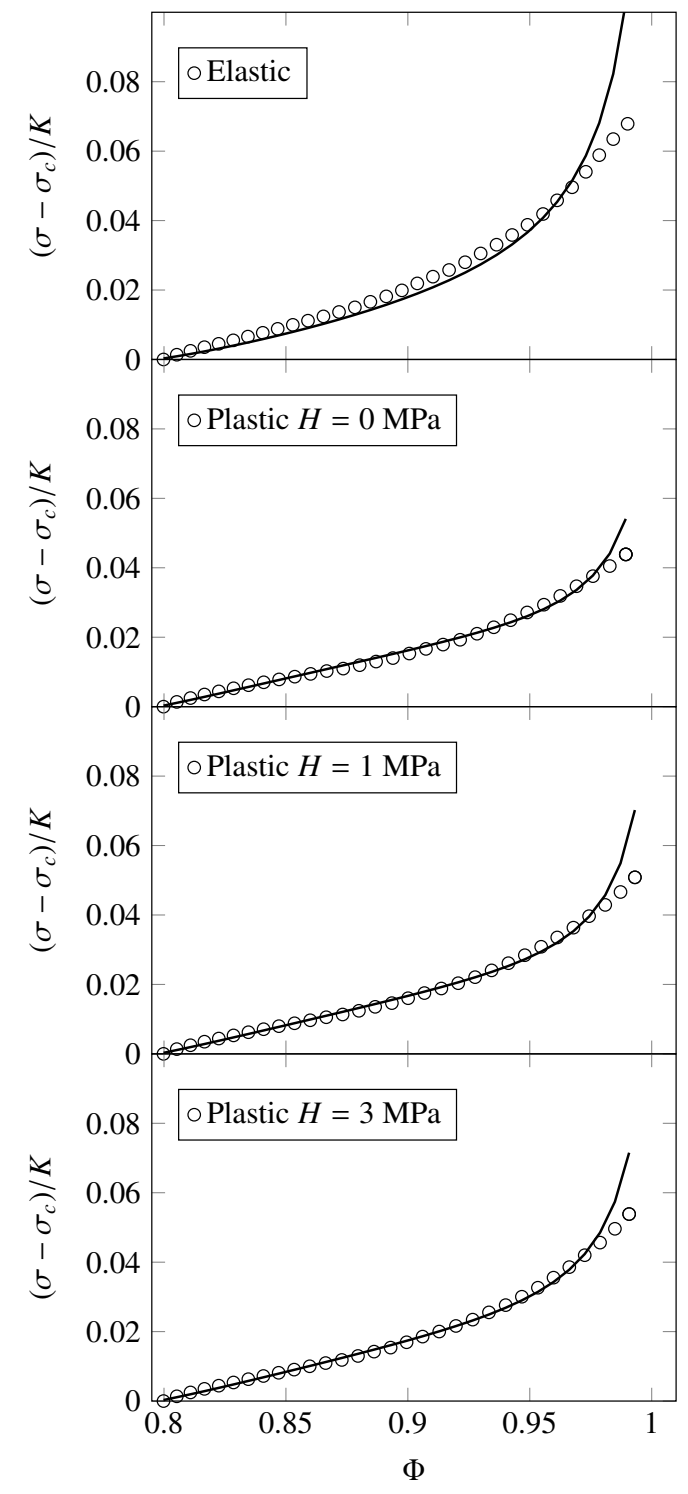

Figure 4. The excess applied stress $\sigma-\sigma_{c}$ normalized by the grain bulk modulus $K$ as a function of packing fraction $\Phi$ for elastic and elasto-plastic grains. $\sigma_{c}$ corresponds to the jamming point, $\Phi_{c} \simeq 0.8$. The lines show the predicted behaviors beyond jamming state by the compaction models introduced in this paper; see Eqs. (8) and (12).

interesting to observe that these models can reproduce well the elasto-plastic grain packing behaviors with the same parameters of the elastic grains (see Sec. 3.1). However, as before, we see a divergence between numerical results and analytical predictions at high packing fractions due to insufficient discretization of grain boundaries.

\section{Conclusions}

In this paper, a discret-continuum approach, the MPM-CD procedure, was used to study the uniaxial compression of deformable granular packings. This numerical approach are coupled the material point method (MPM) with the contact dynamics (CD) method. Two types of material behaviors for grains wre considered: neo-Hookean and elasto-plastic laws. We characterized the packing macroscopic behaviors by the evolution of the packing vertical stress and the packing fraction. Around the jamming state, we observe a linear behavior similar to hard granular systems with a Hertzian contact. However, beyond the jamming state, the packing behaviors are nonlinear. Considering at this state, soft granular packing as a porous continuum media leaded to predictive models able to reproduce well numerical results. These models seem to be quite robust although they still need to be validated by other simulations and experimental results. Moreover, although the considered sample sizes seem not to be large, the effects related to the finite number of particles are almost negligible in the pressure ranges considered in this work [13]. However, this point should be more investigated in future studies.

This work (PaMaCo project) was publicly funded through ANR (the French National Research Agency) under the "JCJC" program. We are also grateful to the Genotoul bioinformatics platform Toulouse Midi- Pyrenees (Bioinfo Genotoul) for providing computing resources.

\section{References}

[1] J.M. Erikson, N.W. Mueggenburg, H.M. Jaeger, S.R. Nagel, Phys. Rev. E 66, 040301 (2002)

[2] R. Bonnecaze, M. Cloitre, Adv. Polym. Sci. 236, 117 (2010)

[3] M. van Hecke, J. Phys. Condens. Matter 22, 033101 (2010)

[4] D. Cantor, M. Cárdenas-Barrantes, I. Preechawuttipong, M. Renouf, E. Azéma, Phys. Rev. Lett. 124, 208003 (2020)

[5] S. Nezamabadi, T. Nguyen, J.Y. Delenne, F. Radjai, Granul. Matter 19, 8 (2017)

[6] S. Nezamabadi, F. Radjai, J. Averseng, J.Y. Delenne, J. Mech. Phys. Solids 83, 72 (2015)

[7] S. Nezamabadi, X. Frank, J.Y. Delenne, J. Averseng, F. Radjai, Comput. Phys. Commun 237, 17 (2019)

[8] T.L. Vu, S. Nezamabadi, S. Mora, Soft Matter 16, 679 (2020)

[9] I. Agnolin, J.N. Roux, Phys. Rev. E 76, 061304 (2007)

[10] J. Kováčik, J. Mater. Sci. Lett. 8, 1007 (1999)

[11] J. Kováčik, J. Mater. Sci. Lett. 20, 1953 (2001)

[12] ANSYS, ANSYS theory reference for the mechanical APDL and mechanical applications, ANSYS Inc., Canonsburg, PA, 12th edn. (2009)

[13] C.P. Goodrich, A.J. Liu, S.R. Nagel, Phys. Rev. Lett. 109, 095704 (2012) 